\title{
BOUNDS FOR SOLUTIONS OF PERTURBED DIFFERENTIAL EQUATIONS
}

\author{
T. G. PROCTOR ${ }^{1}$
}

ABSTRACT. A modified form of the Alekseev variation of constants equation is used to relate the solutions of systems of the form $\dot{x}=f(t, x, \lambda), \lambda$ in $R^{m}$ and the perturbed system $\dot{y}=f(t, y, \psi(t))+g(t, y)$. Hypotheses are given on the $m$ parameter family of differential equations $\dot{x}=f(t, x, \lambda)$ so that if $\dot{\psi}$ and $g$ are perturbation functions, bounds can be calculated for the solutions of the perturbed system.

The purpose of this paper is to study bounds for the solutions of a system of differential equations of a particular form. In the differential equation $\dot{y}=B(t, y)$ we assume $B$ can be written as a sum $B(t, y)=f(t, y, \psi(t))+$ $g(t, y)$ where the $m$-parameter family of differential equations $\dot{x}=f(t, x, \lambda)$ has nice properties and the functions $\dot{\psi}$ and $g$ are perturbation functions. The bounds are established using a variation of constants formula due to V. M. Alekseev [1] in a modified form in much the same way that perturbations of linear systems have been studied using the standard variation of constants formula [3, pp. 64-70]. The bounds are given as solutions of Volterra integral equations using a standard comparison method.

Let $m$ and $n$ be positive integers, let $c>0$, let $S_{c}$ be the closed ball of radius $c$ in $R^{m}$ and let $R^{+}$denote the nonnegative numbers. We assume $f$ and the matrices of derivatives $f_{x}, f$ are continuous for $(t, x, \lambda)$ in $R^{+} \times R^{n} \times$ $S_{c}\left(f(t, x, \lambda)\right.$ in $\left.R^{n}\right), \psi$ is in $C^{1}\left(R^{+}, S_{c}\right)$ and $g$ is in $C\left(R^{+} \times R^{n}, R^{n}\right)$. The solution $\phi(t, \tau, \gamma, \lambda)$ of

$$
\dot{x}=f(t, x, \lambda), \quad x(\tau)=\gamma,
$$

Received by the editors June 4, 1973.

AMS (MOS) subject classifications (1970). Primary 34C10.

Key words and phrases. Perturbed differential equations, Alekseev formula, bounds, comparison theorems, Volterra integral equations.

1 This paper was written while the author, whose research was supported under the National Science Foundation, Grant GP-28931X2, was on sabbatical leave from the Department of Mathematical Sciences, Clemson University, Clemson, South Carolina 29631. 
for $0 \leq \tau \leq t, \gamma$ in $R^{n}$ and $\lambda$ in $S_{c}$ exists for $t$ near $\tau$ as do the matrices of derivatives $\phi_{\gamma}$ and $\phi_{\boldsymbol{\lambda}}[3]$.

The orem 1. Let $0 \leq \tau<t_{0}$ and let $y(t)$ be a solution of

$$
\dot{y}=f(t, y, \psi(t))+g(t, y), \quad y(\tau)=\gamma,
$$

for $\tau \leq t \leq t_{0}$ where $\phi(t, s, y(s), \lambda)$ exists for $\tau \leq s \leq t \leq t_{0^{\circ}}$ Then $y$ satisfies

$$
\begin{gathered}
y(t)=\phi(t, \tau, \gamma, \psi(\tau))+\int_{\tau}^{t} H(t, s, y(s)) d s, \quad \tau \leq t \leq t_{0} \\
H(t, s, y)=\phi_{\gamma}(t, s, y, \psi(s)) g(s, y)+\phi_{\lambda}(t, s, y, \psi(s)) \dot{\psi}(s) .
\end{gathered}
$$

Proof. For $u=(x, \lambda)$ in $R^{n} \times S_{c}$ and $t$ in $R^{+}$the function

$$
\mathcal{F}(t, u)=\operatorname{column}(f(t, x), 0) \text {, }
$$

the matrix of derivatives $\mathcal{F}_{u}$ and the function

$$
\mathcal{S}(t, u)=\operatorname{column}(g(t, x), \dot{\psi}(t))
$$

are continuous. The problem $\dot{u}=\mathcal{F}(t, u), u(\tau)=\operatorname{column}\left(\gamma, \lambda^{*}\right) \equiv \Gamma$ with solution $\Phi(t, \tau, \Gamma)=$ column $\left(\phi\left(t, \tau, \gamma, \lambda^{*}\right), \lambda^{*}\right)$ and the problem

$$
\dot{v}=\mathcal{F}(t, v)+\mathcal{S}(t, v), \quad v(\tau)=\Gamma,
$$

are related by the Alekseev formula [3, Lemma 3, p. 201]

$$
v(t)=\Phi(t, \tau, \Gamma)+\int_{\tau}^{t} \Phi_{\Gamma}(t, s, v(s)) \biguplus(s, v(s)) d s .
$$

Equation (3) follows from (5) when $\lambda^{*}=\psi(\tau)$.

Remark 1. There is a corresponding version of Theorem 1 when $\psi$ depends on $t$ and $y$ (see [8]); however we do not need the more complicated form for the subsequent work.

Remark 2. We recall [3, p. 22] that $\phi_{\lambda}$ satisfies a linear differential equation which gives the representation

$$
\phi_{\lambda}(t, s, y, \lambda)=\int_{s}^{t} \phi_{\gamma}(t, s, y, \lambda) \phi_{\gamma}^{-1}(\nu, s, y, \lambda) f_{\lambda}(v, \phi(v, s, y, \lambda), \lambda) d v .
$$

Equation (3) is a variation of constants type formula which gives a comparison between the solutions of the family of systems (1) and the perturbed system (2). We will use this equation to deduce bounds on the solutions of (3) under appropriate hypotheses. If $A$ is an $n \times n$ matrix we denote by $\mu(A)$ 
its "logarithmic" norm [3]

$$
\mu(A)=\lim _{h \rightarrow 0} \frac{|I+h A|-1}{h}
$$

and make the additional hypotheses:

(i) For $t$ in $R^{+}$and $\lambda$ in $S_{c}$ there are functions $\alpha(t, \lambda)$ and $\beta(t, \lambda)$ such that

$$
\mu\left(f_{x}(t, x, \lambda)\right) \leq \alpha(t, \lambda), \quad \mu\left(-f_{x}^{T}(t, x, \lambda)\right) \leq \beta(t, \lambda)
$$

for $(t, x, \lambda)$ in $R^{+} \times R^{n} \times S_{c}$ where $f_{x}^{T}$ denotes the transpose of $f_{x}$.

(ii) For $(t, \lambda)$ in $R^{+} \times S_{c}, f(t, 0, \lambda)=0$. There is a continuous function $l(t)$ for $t \geq 0$ such that $\left|f_{\lambda}(t, x, \lambda)\right| \leq l(t)|x|$.

(iii) There is a continuous function $h$ from $R^{+}$into $R^{+}$such that

$$
|g(t, x)| \leq h(t)|x|
$$

for $(t, x)$ in $R^{+} \times R^{n}$.

Remark 3. The first inequality in (6) and (ii) imply $\phi(t, s, \gamma, \lambda)$ exists for $0 \leq s \leq t, \gamma$ in $R^{n}, \lambda$ in $s_{c}$ and

$$
|\phi(t, s, \gamma, \lambda)| \leq|\gamma| \exp \int_{s}^{t} \alpha(\tau, \lambda) d \tau, \quad\left|\phi_{\gamma}(t, s, \gamma, \lambda)\right| \leq \exp \int_{s}^{t} \alpha(\tau, \lambda) d \tau .
$$

See [2, pp. 199-200]. If $Z(t)=\left[\phi_{\gamma}^{-1}(t, s, \gamma, \lambda)\right]^{T}$ then $\dot{Z}=-f_{x}^{T}(t, \phi(t, s, \gamma, \lambda), \lambda) Z$ so

$$
\left|\phi_{\gamma}^{-1}(t, s, \gamma, \lambda)\right|=|Z(t)| \leq \exp \int_{s}^{t} \beta(\tau, \lambda) d \tau .
$$

For $0 \leq s \leq t$ let $k(t, s)$ be given by

$$
\begin{aligned}
k(t, s)= & {\left[h(s)+\int_{s}^{t} l(v) \exp \int_{s}^{v}[\beta(u, \psi(s))+\alpha(u, \psi(s))] d u d v|\dot{\psi}(s)|\right] } \\
& \cdot \exp \int_{s}^{t} \alpha(u, \psi(s)) d u .
\end{aligned}
$$

Theorem 2. Let $\delta>0$, let (i)-(iii) be satisfied, and let $\sigma(t)$ be the solution of

$$
\sigma(t)=\delta \exp \int_{\tau}^{t} \alpha(s, \psi(\tau)) d \tau+\int_{\tau}^{t} k(t, s) \sigma(s) d s .
$$

If $y$ is a solution of (2) with $|y(\tau)| \leq \delta$ then $y$ exists for $t \geq \tau \geq 0$ and satisfies $|y(t)| \leq \sigma(t)$. 
Proof. By Theorem 1 and Remark 3 we have if $y$ is a solution of (2) on a maximal interval of existence then $y$ satisfies (3) and

$$
|y(t)| \leq|y(\tau)| \exp \int_{\tau}^{t} \alpha(s, \psi(\tau)) d s+\int_{\tau}^{t} k(t, s)|y(s)| d s
$$

on this interval. By a standard comparison theorem [5] we have $y$ exists for $t \geq \tau$ and $|y(t)| \leq \sigma(t)$

Remark 4. The solution of (8) may be difficult to obta in exactly; how ever upper bounds for the solution of this linear Volterra integral equation can be obtained by means similar to those used in differential equations [6], [7], or by means of the usual method for finding the resolvant. We note that if $\dot{\psi}(s)$ is small (or zero) over much of $R^{+}, k(t, s)$ has approximately the form required to use Gronwall's lemma.

Remark 5. If $\psi$ is a function of $t$ and $y$, i.e. $\psi=\psi(t, y)$ (see Remark 1), or if the bound (7) is replaced by a nonlinear bound in $|y|$ then a corresponding theorem may be obtained over the interval of existence for the solution of the nonlinear analogue of (8) when the integrand in this integral equation is nondecreasing in $\sigma$.

Corollary 1. Let (i)-(iii) be satisfied and assume there are positive constants $M_{1}$ and $M_{2}$ with $M_{2}<1$ such that

$$
\int_{\tau}^{t} \alpha(s, \psi(\tau)) d s \leq M_{1}, \quad \int_{\tau}^{t} k(t, s) d s \leq M_{2}
$$

for all $t \geq \tau \geq 0$. If $y$ is a solution of (2) then $y$ exists for $t \geq \tau$ and $|y(t)| \leq|\bar{y}(\tau)| e^{M} 1 /\left(1-M_{2}\right)$.

Proof. For $\delta=|y(\tau)|$ we obtain for the solution of (8) at any $t \geq \tau$,

$$
\sigma(t) \leq|y(\tau)| e^{M} 1+M_{2} \sup _{\tau \leq s \leq t} \sigma(s) .
$$

If $\sup _{\tau \leq s \leq t} \sigma(s)=\sigma\left(t_{1}\right)$ we have $\sigma\left(t_{1}\right) \leq|y(\tau)| e^{M_{1}} /\left(1-M_{2}\right)$ which gives the result .

When the solutions $\phi(t, \tau, \gamma, \lambda)$ are known hypothesis (i) may be replaced with bounds on $\phi$ and $\phi_{\gamma}$. The most important such case occurs when $f(t, x, \lambda)=A(\lambda) x$. We assume

$\left(i^{*}\right)$ There is a number $M$ and a continuous real valued function $a(\lambda)$ for $\lambda$ in $S_{c}$ such that $|\exp (A(\lambda) t)| \leq M \exp (a(\lambda) t)$ for $\lambda$ in $S_{c}, t \geq 0$.

(ii*) There is a number $N>0$ such that 


$$
\left|\left(\partial a_{i j} / \partial \lambda_{k}\right)(\lambda)\right| \leq N, \quad i, j=1,2, \ldots, n, k=1,2, \cdots, m,
$$

and $\lambda$ in $S_{c^{*}}$. Here the $a_{i j}(\lambda)$ are the elements of $A(\lambda)$.

By (ii*) there is a number $N_{1}$ such that $|(\partial / \partial \lambda)(A(\lambda) x)| \leq N_{1}|x|$ for $\lambda$ in $S_{c}$. Thus

$$
\left|\phi_{\lambda}(t, s, y, \lambda)\right| \leq M^{2} N_{1}(t-s) \exp (a(\lambda)(t-s))|y| .
$$

For $0 \leq s \leq t$, let $K(t, s)$ be given by

$$
K(t, s)=\left[h(s)+|\dot{\psi}(s)| M^{2} N_{1}(t-s)\right] \exp (a(\psi(s))(t-s)) .
$$

The orem 3. Let $\delta>0$, let $f(t, x, \lambda)=A(\lambda) x, g$ satisfy (i*), (ii*), (iii) above and let $\sigma(t)$ be the solution of

$$
\sigma(t)=\delta \exp a(\psi(\tau))(t-\tau)+\int_{\tau}^{t} K(t, s) \sigma(s) d s .
$$

If $y$ is a solution of (2) with $|y(\tau)| \leq \delta, y$ exists for $t \geq \tau$ and $|y(t)| \leq \sigma(t)$.

The proof proceeds exactly as the proof of Theorem 2. There is, of course, a corollary analogous to Corollary 1 in the case $a(\psi(\tau)) \leq 0$ and $\int_{\tau}^{t} K(t, s) d s$ is bounded above by a number $\theta<1$. This corollary and Corollary 1 guarantee the solution $y=0$ of 2 is stable. Similar hypotheses would guarantee uniform stability.

By replacing the function $a(\lambda)$ with a constant, Theorem 1 and the comparison theorem for Volterra integral equations produce an improvement of a result concerning stability given in Coppel [3, p. 117].

Theorem 4. Let $A(t)$ be a $C^{1}$ matrix defined for $t \geq 0$ such that for some $a>0, M \geq 1,|\exp (A(\lambda) t)| \leq M e^{-a t}$ when $t \geq 0, \lambda \geq 0$. If $y(t)$ is a solution of

$$
\dot{y}=A(t) y
$$

then $|\gamma(t)| \leq \sigma(t) e^{-a t}$ for $0 \leq \tau \leq t$ where $\sigma$ is the solution of

$$
\ddot{\sigma}-M^{2}|A(t)| \sigma=0, \quad \sigma(\tau)=M e^{a \tau}|y(\tau)|, \quad \dot{\sigma}(\tau)=0 .
$$

If $|\dot{A}(t)| \leq \beta$, and $Y(t)$ is a fundamental solution of (9) then

$$
\left|Y(t) Y^{-1}(\tau)\right| \leq M \exp (-(\alpha-M \sqrt{ } \beta)(t-\tau))
$$

Proof. We take $m=1, \psi(t)=t$ and $S_{c}=R$ in Theorem 1 , then if $y$ is a solution of (9) 


$$
\begin{aligned}
|y(t)| \leq & M \exp (-\alpha(t-\tau))|y(\tau)| \\
& +\int_{\tau}^{t} M^{2}(t-s) \exp (-\alpha(t-s))\left|A^{\prime}(s)\right||y(s)| d s .
\end{aligned}
$$

Hence $|y(t)| e^{a t} \leq \sigma(t)$ for $t \geq \tau$, where $\sigma$ satisfies

$$
\left.\sigma(t)=M e^{a \tau} \mid y(\tau)\right\rfloor+\int_{\tau}^{t} M^{2}(t-s)\left|A^{\prime}(s)\right| \sigma(s) d s
$$

and thus $\sigma$ satisfies (10).

If $\left|A^{\prime}(t)\right| \leq \beta$ then $|y(t)| e^{a t} \leq \xi(t)$ for $t \geq \tau$, where $\xi$ satisfies

$$
\xi(t)=M e^{a \tau}|y(\tau)|+\int_{\tau}^{t} M^{2} \beta(t-s) \xi(s) d s .
$$

Thus $|y(t)| \leq M \exp (-\alpha(t-\tau)) \cosh M \sqrt{ } \beta(t-\tau)|y(\tau)|$ which implies (11).

Remark 6. The solution $\sigma(t)$ of $(10)$ satisfies

$$
\sigma(t) \leq\left(\sigma^{2}(t)+\dot{\sigma}^{2}(t)\right)^{1 / 2} \leq M e^{a \tau}|y(\tau)| \exp \left(\int_{\tau}^{t} \mu(s) d s\right),
$$

where $\mu(t)=1 / 2\left(\left(M^{4}\left|A^{\prime}(t)\right|^{2}+1\right)^{1 / 2}-M^{2}\left|A^{\prime}(t)\right|\right)$, by [3, Theorem 3, p. 58].

Another way to obtain bounds for the solution of (2) results from the observation that if $z(t)$ satisfies

$$
\dot{z}=G(t, z), \quad z(\tau)=\gamma,
$$

$$
G(t, z)=\phi_{\gamma}^{-1}(t, \tau, z, \psi(t))\left[g(t, \phi(t, \tau, z, \psi(t)))-\phi_{\lambda}(t, \tau, z, \psi(t)) \dot{\psi}(t)\right],
$$

then $y=\phi(t, \tau, z(t), \psi(t))$ satisfies (2). A similar study was given in [4] for the case when $f$ did not depend on the parameter $\lambda$. Obvious modifications can be made to the hypotheses and conclusions of the theorems of [4] to deduce bounds for the study of (2). Again this method can be altered for the case when $\psi=\psi(t, y)$ depends on $y$; however the results require very complicated hypotheses.

\section{REFERENCES}

1. V. M. Alekseev, An estimate for the perturbations of solutions of ordinary differential equations, Vestnik Moskov. Univ. Ser. I Mat. Meh. 1961, no. 2, 28-36. (Russian) MR 23 \#A2596.

2. F. Brauer, Perturbations of nonlinear systems of differential equations, J. Math. Anal. Appl. 14 (1966), 198-206. MR 33 \#359.

3. W. A. Coppel, Stability and asymptotic behavior of differential equations, Heath, Boston, Mass., 1965. MR $32 \# 7875$. 
4. R. E. Fennell and T. G. Proctor, Perturbations of nonlinear differential equations, Trans. Amer. Math. Soc. 185 (1973), 401-411.

5. R. K. Miller, Nonlinear Volterra integral equations, Benjamin, Menlo Park, California, 1971.

6. B. Noble, The numerical solution of nonlinear integral equations and related topics, Nonlinear Integral Equations (Proc. Advanced Seminar Conducted by Math. Research Center, U. S. Army., Univ. Wisconsin, Madison, Wis., 1963), Univ. Wisconsin Press, Madison, Wis., 1964, pp. 215-318. MR 30 \#3582.

7. J. A. Nohel, Some problems in nonlinear Volterra integral equations, Bull. Amer. Math. Soc. 68 (1962), 323-329. MR 26 \#2838.

8. T. G. Proctor, Periodic solutions for perturbed differential equations, J. Math. Anal. Appl. (to appear).

CENTER FOR DYNAMICAL SYSTEMS, DIVISION OF APPLIED MATHEMATICS, BROWN UNIVERSITY, PROVIDENCE, RHODE ISLAND 02912

Current address. Department of Mathematical Sciences, Clemson University, Clemson, South Carolina 29631 